\title{
Retraction: Seokbin Lim. Steady State Analytical Equation of Motion of Linear Shaped Charges Jet Based on the Modification of Birkhoff Theory. Appl. Sci. 2012, 2, 35-45
}

\section{Seokbin Lim}

Energetic Systems Research Group, Department of Mechanical Engineering, New Mexico Tech, Socorro, NM 87801, USA; E-Mail: lim@nmt.edu; Tel.: +1-575-835-6589; Fax: +1-575-835-5209

Received: 27 October 2013; in revised form: 3 December 2013 / Accepted: 4 December 2013 /

Published: 17 December 2013

The following article: doi:10.3390/app2010035 [1] has been retracted by the author due to problems in the hydrocode simulation configuration.

The paper aims to develop an analytical approach in calculation of the jet/slug velocity of typical linear shaped charges (LSCs) based on a rather simple modification of the conventional Birkhoff theory. In the context, the author uses a simple 2D simulation to prove the accuracy of the analytical calculation and to identify general characteristics of the proposed analytical method. However, later it was found that the author ignored the unique geometrical configuration of LSCs in simulations (properly addressable in 3D configuration), creating a questionable comparison between the analytical calculation and the simulation result; causing the retraction of the paper [1]. The author, however, confirmed that the analytical calculation does not contain any flaws. As an author, I take full responsibility for the retraction and any other errors in its contents.

\section{Reference}

1. Lim, S. Steady State Analytical Equation of Motion of Linear Shaped Charges Jet Based on the Modification of Birkhoff Theory. Appl. Sci. 2012, 2, 35-45.

(C) 2013 by the authors; licensee MDPI, Basel, Switzerland. This article is an open access article distributed under the terms and conditions of the Creative Commons Attribution license (http://creativecommons.org/licenses/by/3.0/). 